\title{
Thermal analysis of lithium-ion batteries with square frame geometries by theoretical
}

simulations

D. Miranda, F. Miranda, C. M. Costa, A. M. Almeida, and S. Lanceros-Méndez

Citation: AIP Conference Proceedings 1863, 440005 (2017);

View online: https://doi.org/10.1063/1.4992609

View Table of Contents: http://aip.scitation.org/toc/apc/1863/1

Published by the American Institute of Physics

\section{Articles you may be interested in}

Evaluation and optimization of the performance of frame geometries for lithium-ion battery application by computer simulation

AIP Conference Proceedings 1738, 370010 (2016); 10.1063/1.4952155 


\title{
Thermal Analysis of Lithium-Ion Batteries with Square Frame Geometries by Theoretical Simulations
}

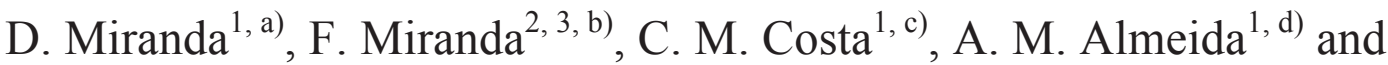 \\ S. Lanceros-Méndez ${ }^{1,4,5, \text { e) }}$ \\ ${ }^{1}$ Centro/Departamento de Física, Universidade do Minho, 4710-057 Braga, Portugal \\ ${ }^{2}$ CIDMA, Universidade de Aveiro, Aveiro, Portugal \\ ${ }^{3}$ Instituto Politécnico de Viana do Castelo, Viana do Castelo, Portugal \\ ${ }^{4}$ BCMaterials, Parque Científico y Tecnológico de Bizkaia, 48160-Derio, Spain \\ ${ }^{5} I K E R B A S Q U E$, Basque Foundation for Science, Bilbao, Spain \\ ${ }^{a)}$ Corresponding author: danielmir.fis@gmail.com \\ b)fmiranda@estg.ipvc.pt \\ c)cmscosta@fisica.uminho.pt \\ d)coimbra@fisica.uminho.pt \\ e)lanceros@fisica.uminho.pt
}

\begin{abstract}
Thermal analysis of battery geometries is essential for applications, as the thermal behavior of a battery influences its cycling life. Two geometries, square frame and conventional, have been studied for a scan rate of 290C. The square frame shows a capacity value of $290,15 \mathrm{Ahm}^{-2}$ which is 446 times higher than the one for the conventional geometry, when the area is maintained constant for all components. In the adiabatic model, the square frame geometry has higher capacity value in comparison to the conventional geometry.
\end{abstract}

Keywords: Thermal analysis, simulation, capacity, lithium ion battery.

PACS: 88.80.F-, 88.80.ff, 88.05.-b

\section{INTRODUCTION}

Efficient energy storage systems such as lithium ions batteries [1] are essential for many applications including mobile-phones, computers, e-labels, e-packaging and disposable medical testers, among others, as well as for hybrid electric vehicles (PHEVs) or electric vehicles (EVs), being necessary high energy and power without forgetting the environmental issues [2].

The components of lithium-ion batteries are the anode (negative electrode), the cathode (positive electrode) and the separator/electrolyte, the materials determining energy and power [2].

Other possibility for increasing the performance of lithium-ion batteries is by varying its geometry.

The influence of non-conventional geometries (horseshoe, spiral, ring, antenna and gear batteries) in the performance of lithium-ion batteries has been demonstrated [3].

Further, it was verified that a square frame shows a capacity value of $305,52 \mathrm{Ahm}^{-2}$, which is 527 times higher than the one for a conventional geometry, while maintaining the area of the different components [4].

One factor that affects the performance of a battery is the temperature, the thermal management allowing to improve the life of the battery [5].

Taking into account that the combination of geometry and thermal analysis is a key for obtain high capacity, the goal of this work is to analyze the thermal behavior of a square frame geometry in comparison with a conventional geometry. The thermal analysis has been performed at different scan rates through finite element method simulation maintaining a constant area for the different components. In the square frame geometry, the thermal behavior was carried out taking into account the length of the side.

These geometries are illustrated in Figures 1 (a) and (b) for the conventional and the square frame geometries, respectively.

Finite Elements analysis were carried for each geometry through of the Doyle/Fuller/Newman, the equations of the model describing the physical and electrochemical phenomena that occur along the operation of batteries in two dimensions [6-7].

International Conference of Numerical Analysis and Applied Mathematics (ICNAAM 2016)

AIP Conf. Proc. 1863, 440005-1-440005-5; doi: 10.1063/1.4992609

Published by AIP Publishing. 978-0-7354-1538-6/\$30.00 
For the evaluation of the effect of the geometry in the thermal behaviour, the area was maintained constant for the different components of the batteries (electrodes, separator and current collectors).

Further, in the square geometry, the variable L is defined as the length of the side, as illustrated in Figure 1 (b).

The square frame geometry is adequate for portable devices such as, smart-phones, tablets and portable computers, as the empty space allows integration of the electronic components at the center of the battery.

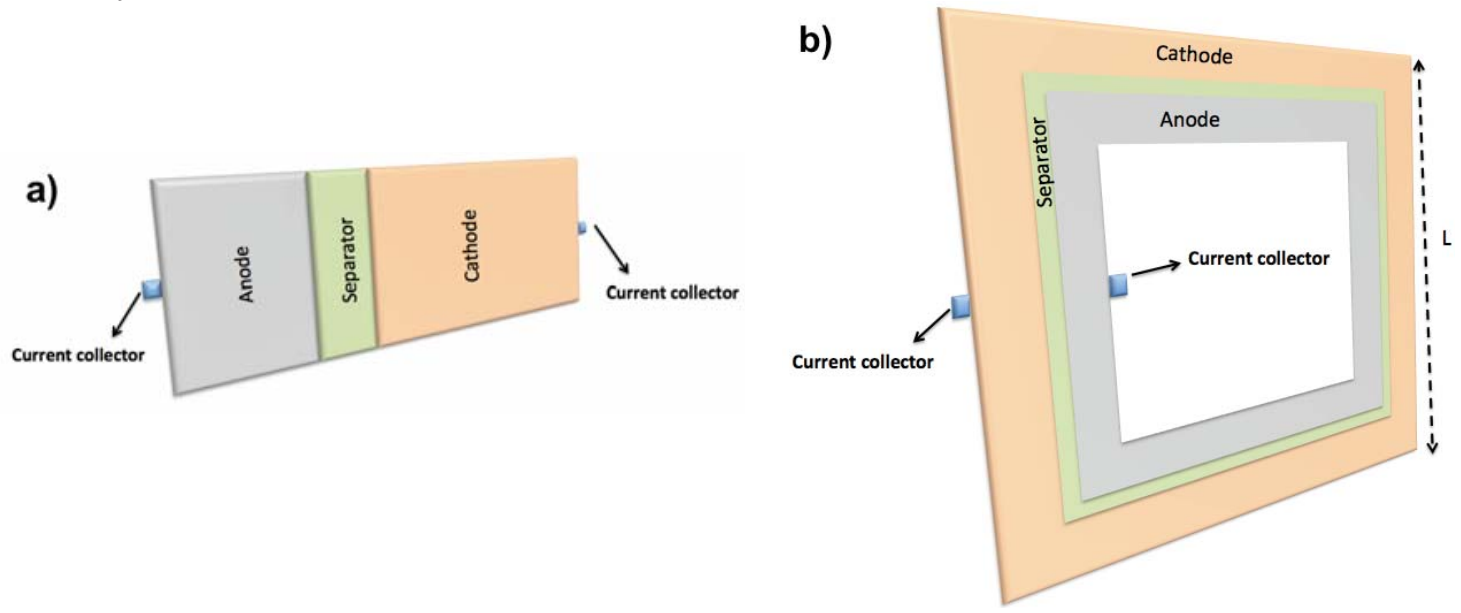

FIGURE 1. Geometries implemented in the theoretical simulations (a) conventional geometry and (b) square frame geometry.

First, the capacity value of the two geometries at low, medium and high discharge rates (from $1 \mathrm{C}$ up to 500C) was evaluated applying isothermal and adiabatic models with initial temperature of $298 \mathrm{~K}$ and length of side in the square frame geometry of $100 \mu \mathrm{m}$.

Then, the influence of the side length of the square frame geometry was evaluated between $20 \mu \mathrm{m}$ to $600 \mu \mathrm{m}$ at $500 \mathrm{C}$ scan rate in isothermal and adiabatic conditions.

Table 1 shows the parameters used for the theoretical simulations of the different geometries.

\begin{tabular}{|c|c|c|c|c|}
\hline Parameter & Unit & Anode $\left(\mathrm{Li}_{\mathrm{x}} \mathrm{C}_{6}\right)$ & Separator & Cathode $\left(\mathrm{Li}_{\mathrm{x}} \mathrm{Mn}_{2} \mathrm{O}_{4}\right)$ \\
\hline$C_{E, i, 0}$ & $\mathrm{~mol} / \mathrm{m}^{3}$ & 14870 & & 3900 \\
\hline$C_{E, i, \max }$ & $\mathrm{mol} / \mathrm{m}^{3}$ & 26390 & & 22860 \\
\hline$C_{L}$ & $\mathrm{~mol} / \mathrm{m}^{3}$ & & 1000 & \\
\hline$r$ & $\mathrm{~m}$ & $12,5 \times 10^{-6}$ & & $8 \times 10^{-6}$ \\
\hline $\boldsymbol{L}_{i}$ & $\mathrm{~m}$ & $200 \times 10^{-6}$ & $90 \times 10^{-6}$ & $400 \times 10^{-6}$ \\
\hline $\boldsymbol{k}_{i}$ & $\mathrm{~S} / \mathrm{m}$ & $\left(6,5 \times 10^{-1}\right) \times 0,357^{1,5}$ & $\left(6,5 \times 10^{-1}\right) \times 4,84 \times 10^{-2}$ & $\left(6,5 \times 10^{-1}\right) \times 0,444^{1,5}$ \\
\hline$D_{i}$ & $\mathrm{~m}^{2} / \mathrm{s}$ & $\left(D_{a}\right) \times 0,357^{1,5}$ & $\left(4,0 \times 10^{-10}\right) \times 4,84 \times 10^{-2}$ & $\left(D_{p}\right) \times 0,444^{1,5}$ \\
\hline$D_{L I}$ & $\mathrm{~m}^{2} / \mathrm{s}$ & $3,9 \times 10^{-14}$ & & $1 \times 10^{-13}$ \\
\hline Brugg or $p$ & & 1,5 & 8,5 & 1,5 \\
\hline$\varepsilon_{f, i}$ & & 0,172 & & 0,259 \\
\hline$\varepsilon_{i}$ & & 0,357 & 0,70 & 0,444 \\
\hline$\tau$ & & & 3,8 & \\
\hline$\sigma_{i}$ & $\mathrm{~S} / \mathrm{m}$ & 100 & & 3,8 \\
\hline$i_{I C}$ & $\mathrm{~A} / \mathrm{m}^{2}$ & & 17,5 & \\
\hline$T$ & $\mathrm{~K}$ & & 298,15 & \\
\hline T_ref_a & K & 318 & & \\
\hline T_ref_p & K & & & 298,15 \\
\hline$A_{i}$ & $\mathrm{~m}^{2}$ & $4,0 \times 10^{-8}$ & $1,8 \times 10^{-9}$ & $8,0 \times 10^{-8}$ \\
\hline$r$ & $\mathrm{~kg} / \mathrm{m}^{3}$ & 2270 & 1008.98 & 2500 \\
\hline $\boldsymbol{K}$ & $\mathrm{W} /(\mathrm{m} . \mathrm{K})$ & 1 & 0.344 & 1.58 \\
\hline$C p$ & $\mathrm{~J} /(\mathrm{kg} . \mathrm{K})$ & 881 & 1978.16 & 1269.21 \\
\hline
\end{tabular}

Auxiliary equations:

Anode: $\mathrm{D}_{\mathrm{a}}=1.4523 \mathrm{e}^{-13 *} \exp \left(68025.7 / 8.314 *\left(1 /\left(\mathrm{T} \_r e f \_\mathrm{a} / 1\right)-1 /\left(\mathrm{T} \_2 \_\mathrm{a} / 1\right)\right)\right)\left[\mathrm{m}^{2} / \mathrm{s}\right], \mathrm{T} \_2 \_\mathrm{a}=$ $\min (393.15, \max (\mathrm{T}, 223.15))$

Cathode $: \mathrm{D}_{\mathrm{p}}=1 \mathrm{e}^{-14 *} \exp \left(20000 / 8.314 *\left(1 /\left(\mathrm{T} \_r e f \_\mathrm{p} / 1\right)-1 /\left(\mathrm{T} \_2 \_\mathrm{p} / 1\right)\right)\right)\left[\mathrm{m}^{2} / \mathrm{s}\right], \mathrm{T} \_2 \_\mathrm{p}=$ $\min (393.15, \max (\mathrm{T}, 223.15))$ 


\section{RESULTS AND DISCUSSION}

Figure 2 (a) shows the delivered discharge capacity values obtained as a function of the scan rate for conventional and square frame geometry with side length of $100 \mu \mathrm{m}$ applied within an isothermal model at constant temperature of $298 \mathrm{~K}$.
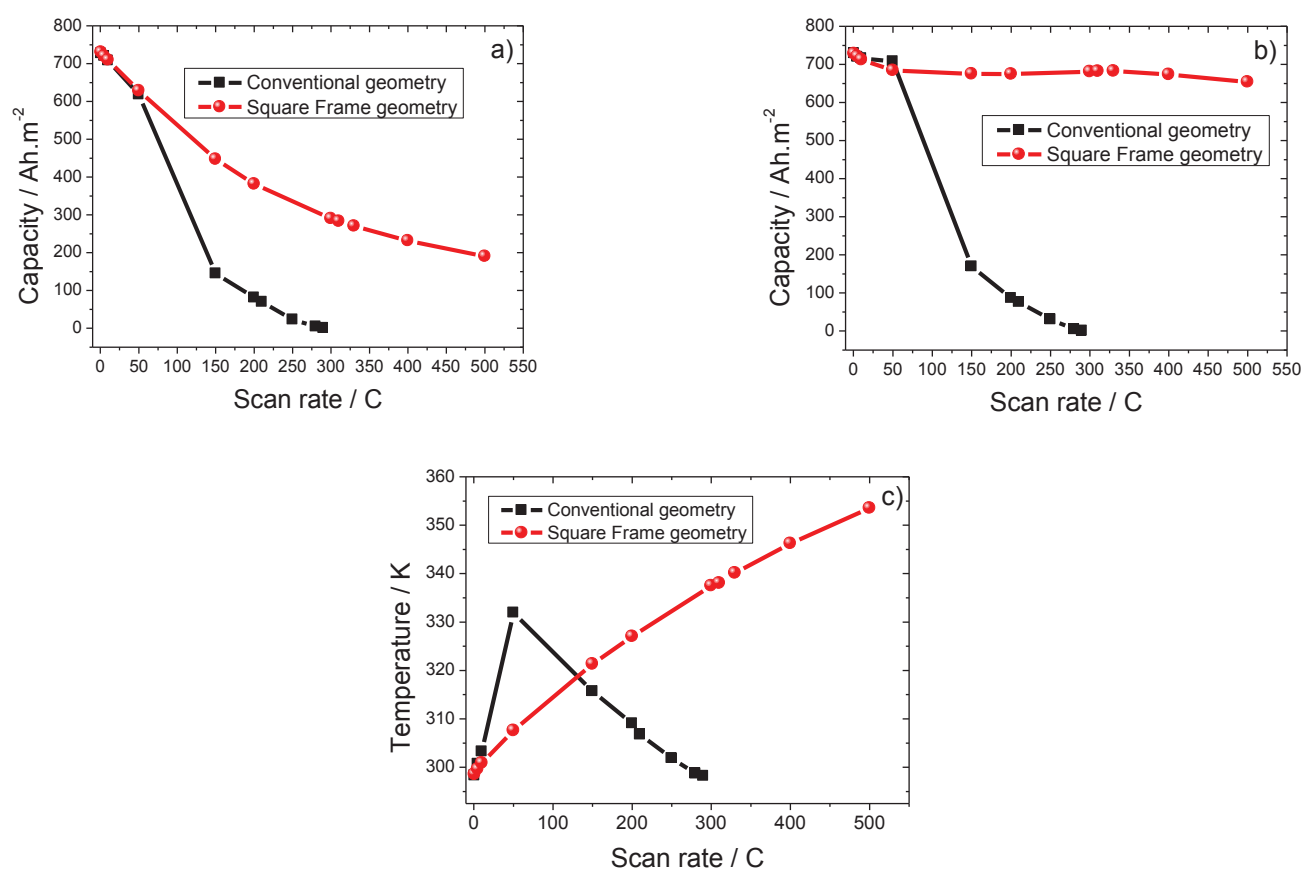

FIGURE 2. Delivered capacity as a function of scan rate for conventional and square frame geometries in the isothermal model at $298 \mathrm{~K}$ (a), and the adiabatic model with initial temperature of $298 \mathrm{~K}$ (b). Temperature in the adiabatic model for both geometries as a function of scan rate (c).

The cycling results (Figure 2 (a)) for the isothermal model shows that the square frame geometry has a higher performance in comparison to the conventional geometry independently of the scan rate.

In the isothermal model (Figure 2 (a)), the conventional geometry shows a capacity of $0.65 \mathrm{Ah} \cdot \mathrm{m}^{-2}$ at $290 \mathrm{C}$ and the square frame geometry shows a capacity value of $290.15 \mathrm{Ah}^{-2} \mathrm{~m}^{-2}$ at the same scan rate: 446 times higher than the conventional geometry. It is also observed that the conventional geometry does not work for scan rates above $290 \mathrm{C}$, but on the other hand, the square frame geometry can operate at rates higher than $500 \mathrm{C}$. The square frame geometry with a thin separator and a larger contact surface between the electrodes has a larger ionic flow between the electrodes and a higher ion insertion capacity at the cathode, leading to a higher delivered capacity in comparison with the conventional geometry.

Figure 2 (b) shows the delivered capacity values obtained at different scan rates for the conventional and the square frame geometries applying a thermal model in adiabatic conditions with an initial temperature of $298 \mathrm{~K}$. The conventional geometry (Figure 2 (b)) presents the same behavior as the isothermal situation (Figure 2 (a)) at all scan rates

The only difference is that for each scan rate, the discharge capacity value is higher for the adiabatic situation in comparison to the isothermal situation.

This fact is due to the increase of the battery temperature (as a result of the heat generated in the battery) which leads to higher ionic conductivity and diffusion, increasing the capacitance value as it is shown in Figure 2 (c). In the conventional battery, the increase of scan rate implies a decrease of its capacity and also of the discharge time.

The conventional geometry can operate at all rates from $1 \mathrm{C}$ to $290 \mathrm{C}$, excluding the rate of $50 \mathrm{C}$. At $50 \mathrm{C}$ the battery generates a high amount of heat that will be absorbed, which combined with long discharging time allows a significant increase in bulk temperature. Thus, the battery reaches a temperature above of degradation temperature of the materials. Above $50 \mathrm{C}$, the battery delivers larger amounts of heat, but at these rates the discharge time is much lower, short enough not to reach the degradation temperature.

For the square frame geometry with $\mathrm{L}=100 \mu \mathrm{m}$ it can operate at all discharge rates from $1 \mathrm{C}$ to $150 \mathrm{C}$, but above $150 \mathrm{C}$ the battery reaches a temperature above the degradation temperature. 
Figure 3 (a) shows the discharge capacity value of the square frame geometry for different dimensions of the side length at $500 \mathrm{C}$ in the isothermal and the adiabatic model. The size range is set between $20 \mu \mathrm{m}$ to $600 \mu \mathrm{m}$.

In both thermal models, it is found that with increasing side length dimension from $20 \mu \mathrm{m}$ to $100 \mu \mathrm{m}$, the discharge capacity value increases. For $100 \mu \mathrm{m}$ to $600 \mu \mathrm{m}$, the discharge capacity value decreases, the difference lying in the fact that for the square frame geometry in the adiabatic model has much higher capacity values that the square frame geometry in the isothermal model, as the heat produced in the adiabatic model allows a significant increase in temperature and hence an increase of the diffusion and ion conductivity.
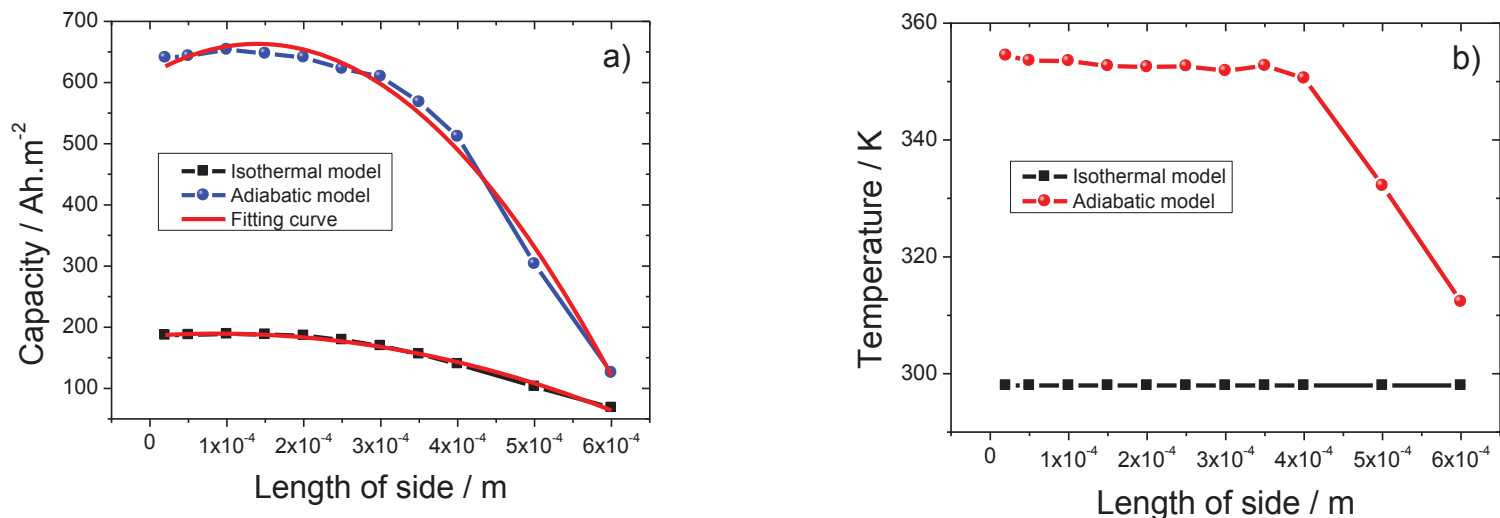

FIGURE 3. Delivered capacity (a) and temperature (b) as a function of side length of the square frame geometry at $500 \mathrm{C}$ for isothermal and adiabatic models. Fitting of the capacity values as a function of side length through a Quadratic Regression Polynomial using Least Square Method.

Using least square method, the optimal length side is $84 \mu \mathrm{m}$ and $120 \mu \mathrm{m}$ for the isothermal and adiabatic model, respectively. The differences are due to the temperature of the adiabatic conditions as is shown in Figure 3 (b).

\section{CONCLUSION}

The combination of battery geometry with the thermal management is essential to improve the cycle life and also its safety. In this work, the capacity value of the conventional and square frame geometries is evaluated in isothermal and adiabatic conditions. When the geometrical parameter (side length of a square frame) is optimized according to the area, high discharge capacity values are obtained with respect to a conventional geometry. At $290 \mathrm{C}$, the square frame geometry shows a capacity value of $290,15 \mathrm{Ahm}^{-2}$ with length side of $100 \mu \mathrm{m}$ which is 446 times higher than the one for the conventional geometry for constant area of all components.

For the square frame geometry at $500 \mathrm{C}$, the optimal length side is $84 \mu \mathrm{m}$ and $120 \mu \mathrm{m}$ for isothermal and adiabatic conditions, respectively.

\section{ACKNOWLEDGMENTS}

This work was supported by the Portuguese Foundation for Science and Technology (FCT) in the framework of the Strategic Funding UID/FIS/04650/2013. The authors thank FEDER funds through the COMPETE 2020 Programme and National Funds through FCT - Portuguese Foundation for Science and Technology under the projects PTDC/CTM-ENE/5387/2014 and UID/CTM/50025/2013 and grants SFRH/BPD/112547/2015 (C.M.C.). SLM thanks financial support from the Basque Government Industry Department under the ELKARTEK Program. F. Miranda was also supported by Portuguese funds through the CIDMA - Center for Research and Development in Mathematics and Applications, and the Portuguese Foundation for Science and Technology ("FCT - Fundação para a Ciência e a Tecnologia"), within project UID/MAT/04106/2013. 


\section{REFERENCES}

1. M. Wakihara, O. Yamamoto, Lithium ion batteries: fundamentals and performance, Kodansha, 1998.

2. B. Scrosati, J. Garche, J Power Sources, 195 (2010) 2419-2430.

3. D. Miranda, et al., Applied Energy, 165 (2016) 318-328.

4. D. Miranda, et al., AIP Conference Proceedings, 1738 (2016) 370010.

5. D. H. Jeon, Current Applied Physics, 14 (2014) 196-205.

6. $\quad$ D. Miranda, et al., Journal of Eletroanalytical Chemistry, 739 (2015) 97-110.

7. M. Doyle, et al., J. Electrochem. Soc., 143 (1996) 1890-1903. 